\title{
Antibacterial and molecular dynamics study of the Dolabellanin B2 isolated from sea slug, Peronia peronii
}

\author{
M.A. Bitaab ${ }^{1 *}$, S.O. Ranaei Siadat ${ }^{2}$, J. Pazooki ${ }^{1}$ and Y. Sefidbakht ${ }^{2}$ \\ ${ }^{1}$ Department of Marine biology, Faculty of Biological Science, \\ Shahid Beheshti University G.C. Evin, Tehran, Iran. \\ ${ }^{2}$ Protein Research Center, Shahid Beheshti University, G.C. Evin, Tehran, Iran.
}

DOI: http://dx.doi.org/10.13005/bbra/1870

(Received: 26 July 2015; accepted: 03 October 2015)

\begin{abstract}
Marine gastropod, Peronia peronei, from north of the Persian Gulf, protein extraction was investigated using ultrafiltration, reversed-phase high-performance liquid chromatography and matrix-assisted laser desorption/ionization time-of-flight mass spectrometry (MALDI-TOF/TOF). The mass spectra showed that, the isolated peptide is most probably Dolabellanin B2 that has been extracted before from other marine mollusk, Dolabella auricularia. The antibacterial activity of purified peptide was assessed against seven bacterial strains (Staphylococcus aureus, Bacillus subtilis, Serratia marcescens, Escherichia coli, Klebsiella pneumoniae, Pseudomonas aeruginosa and Proteus mirabilis). Broth micro-dilution method, standard disk diffusion assay and bioautography were used for this aim. According to the results the identified peptide showed strong antibacterial activity against all mentioned bacterial strains. Computational studies through peptide modeling servers followed by $\mathbf{5 0} \mathrm{ns}$ molecular dynamics simulation and surface electrostatic calculations indicates that this sequence could be most probably related to alpha helical cationic antimicrobial peptides (alpha-CAMPs) with overall charge of +1 at $\mathrm{pH} 7$.
\end{abstract}

Key words: Antibacterial peptide, Peronia peronii, Dolabellanin B2, molecular dynamics

Marine ecosystems cover $71 \%$ of the earth's surface and contain tremendous species richness and approximately half of the total biodiversity. During the last three decades, marine invertebrates have provided key structure and compounds that proved their potential in various fields, particularly as new therapeutics for a variety of diseases ${ }^{1}$. Natural products derived from marine organisms have become an increasingly important source of biologically active compounds. Marine organisms are seen as rich sources of potential drugs and useful chemicals from nature ${ }^{2}$. The interest in this field is reflected by the number of

\footnotetext{
* To whom all correspondence should be addressed.
}

publications, the variety of novel structures, and different types of organisms investigated ${ }^{2}$.

Infectious diseases caused by bacteria are a major threat to public health. Their impact is more important in developing countries due to the relative unavailability of medicines and the emergence of widespread drug resistance. Most of antimicrobials that used clinically are naturally produced or resemble and mimic natural products. For example, in the twelve antibacterial classes, ninth of them are derived from a natural product template. Molecular architectures of the $\beta$-lactams (carbapenems, monobactams, cephalosporins, and penicillins), polyketides (tetracycline), phenylpropanoid (chloramphenicol), aminoglycosides (streptomycin), glycopeptides (vancomycin), macrolides (erythromycin), 
streptogramins (pristinamycin) and, most newest, the lipopeptides (daptomycin) and glycylcyclines (tegicycline) have been driven from natural products $^{3}$. Other three classes, sulfonamides, quinolones (ciprofloxacin) and oxazolidinones (linezolid), have not detected from natural sources yet $^{4}$. Multi-drug resistant bacteria are also emerging problem in the health care system worldwide. Thus, There has been considerable attention drawn to develop new antibacterial compounds, with novel mechanisms of action that could potentially evade the emergence of drug resistant bacteria ${ }^{5}$. Antimicrobial peptides (AMPs) have an important role in the innate immune system of animals, where they participate in the neutralization and elimination of intruding microorganisms and remained potent for millions of years ${ }^{6}$. They are part of the humoral natural defense of invertebrates against infections that termed "natural antibiotics" ${ }^{7}$. One of the main solutions to the antibiotic resistance is the discovery of novel antimicrobial compounds for clinical applications. In comparison to the terrestrial environment, which has been under focus of the pharmaceutical industry for more than 50 years, aquatic ecosystems have remained virtually unexplored for their pharmacological metabolites ${ }^{4}$. During the last decades, these kinds of antibiotics have attracted attention because of their role as therapeutics or drug leads ${ }^{8-10}$. Most important components of the innate immune defense system in marine invertebrates are antimicrobial peptides. They are molecules mostly less than $10 \mathrm{kDa}$ in mass $^{8}$. The majority of AMPs shares two features that enable them to interact with microbes: i) they have a net positive charge, enables them to interact with membranes of bacteria through electrostatic forces. ii) They also can form amphipathic structures in hydrophobic environments and thus penetrate into the bacterial phospholipid bilayer membrane ${ }^{9}$. Differences in the lipid composition between prokaryotic and eukaryotic cell membranes demonstrate the targets for these peptides. Antimicrobial specificity of AMPs towards the targets (bacteria or fungi) was highly dependent on the preferential interaction of peptides with the microbial cells that enable them to kill target cells without affecting the host cells ${ }^{10}$. Antimicrobial peptides (AMPs) driven from marine sources have been mainly investigated in economically important species like aquaculture species $^{11}$.

Peronia peronii is an air breathing sea slug species and shell-less marine pulmonate gastropod mollusk from Onchidiidae family. The members of this family mostly inhabit the rocky intertidal zones ${ }^{12}$. Generally they can be found in the Persian Gulf, where feeding on algae and encrusting diatoms that attached to the rocks around them.

The present study evaluated the antibacterial activity of Peronia peronii peptide purified by ultrafiltration, HPLC and recognized with time-of-flight mass spectrometry against seven different bacterial strains. The antibacterial activity against Staphylococcus aureus, Bacillus subtilis, Serratia marcescens, Escherichia coli, Klebsiella pneumoniae, Pseudomonas aeruginosa and Proteus mirabilis were studied through broth micro-dilution, standard disk diffusion assay and bio-autography methods. Molecular dynamics studies allow for a detailed classical mechanics description of antibacterial peptides, including sampled structures and their populations, types of motions, geometrical, energetic, thermodynamic, and kinetic description of the system ${ }^{13}$. The 3-D structure of the peptide was investigated using Gromacs 4.6.7 molecular dynamic tools. Electrostatic and surface properties were also analyzed.

\section{MATERIALSANDMETHOD}

\section{Sample collection and Identification}

Marine gastropod, Peronia peronii (Figure 1) was collected from the intertidal zone in south of the Queshm Island in the Persian Gulf $\left(26^{\circ} 40^{\prime}, 58.95^{\circ} \mathrm{N}, 55^{\circ} 43^{\prime}, 10.49^{\circ} \mathrm{E}\right)$ in May 2012. Total 60 marine gastropods were collected (large diameter range 42 to $85 \mathrm{~mm}$ ). All specimens were identified based on the external morphology, shape, and pigmentation features, according to the keys and references ${ }^{14,15}$. The samples were washed extensively with sea water then placed in polyethylene plastic barrels and kept frozen at $80^{\circ} \mathrm{C}$ for future studies after measuring the weight and length.

\section{Extraction}

Extraction was carried out according to Lehrer et al. ${ }^{16-19}$. At first, Whole body (except 
viscera) was ground into fine powder in the liquid nitrogen. The frizzed powder was homogenized with acetic acid/water (5/95; v/v) and stirred for $2 \mathrm{~h}$ at $4{ }^{\circ} \mathrm{C}$. Then the extract was centrifuged at $10,000 \mathrm{~g}$ for $30 \mathrm{~min}$ and the supernatant was recovered and filtrated by an Amicon Ultra $15 \mathrm{ml}$ centrifugal membrane filter device (Merck Millipore) with a 10 KD cut off. Filtered liquid was concentrated 50fold by lyophilizition (Christ Alpha 2-4 LSC plus machine, Germany) followed by salvation in sterile ultrapure water.

\section{Isolation and Identification}

Extracted peptides were separated using reverse-phase HPLC on C18 column $(0.4 \mathrm{~cm} \times 25$ $\mathrm{cm}, 5 \mu \mathrm{m}$ particle sizes, $300 \AA$ pore size) with Knauer HPLC system (Germany) equipped with auto sampler. The flow rate was $1 \mathrm{ml} / \mathrm{min}$ and the mobile phase was water and acetonitrile with $0.1 \%$ TFA (Trifluoroacetic acid). A gradient program was applied (0-20\% acetonitrile). All peaks were monitored at $280 \mathrm{~nm}$ (Knauer UV/Vis detector) which is suitable for peptide and protein. Isolated peaks were collected in separate containers and used for antibacterial tests. Only one fraction has an antibacterial activity and used for further studies.

\section{MALDI-TOF/TOF Mass Spectroscopy}

Positive-ion MALDI mass spectra were obtained using a Bruker ultraflex III (USA) in reflectron mode, equipped with a Nd:YAG smart beam laser. MS spectra were acquired over a mass range of $\mathrm{m} / \mathrm{z}$ 800-4000. Final mass spectra were externally calibrated against an adjacent spot containing 6 peptides (des-Arg1-Bradykinin, 904.681; Angiotensin I, 1296.685; Glu1Fibrinopeptide B, 1750.677; ACTH (1-17 clip), 2093.086; ACTH (18-39 clip), 2465.198; АCTH (738 clip), 3657.929). Monoisotopic mass was obtained using a SNAP averaging algorithm (C 4.9384, N 1.3577, O 1.4773, S 0.0417, H 7.7583) and an $\mathrm{S} / \mathrm{N}$ threshold of 2 [20]. Data were submitted to the Mascot program (Matrix Science, version 2.4) to identify the peptide sequences from the database.

\section{Protein concentration determination}

The samples were dissolved in $50 \mathrm{mM}$ Tris buffer ( $\mathrm{pH}$ 7.0) then protein concentration was measured according to the Pierce Modified Lowry Assay (Thermo Fisher Scientific, Rockford, IL, USA) as well as SDS page densitometry ${ }^{21}$. BSA
(Bovine Serum Albumin) was used for preparing the standard curve. Tricine-SDS-PAGE was performed to estimate the molecular weight of proteins.

\section{Minimum Inhibition Concentration test}

Antibacterial susceptibility tests were performed by the broth micro-dilution method for determination of the MIC (Minimum Inhibitory Concentration), as recommended by CLSI (Clinical and Laboratory Standards Institute) with some minor modifications ${ }^{22}$. Samples were prepared according to their total protein concentrations and using sterile $50 \mathrm{mM}$ Tris buffer $\mathrm{pH} 7.0$ and tested against a collection of standard bacterial strains. The bacteria used in the present investigation were; Staphylococcus aureus, Bacillus subtilis, Escherichia coli, Klebsiella pneumoniae, Proteus mirabilis, Serratia marcescens, and Pseudomonas aeruginosa. $18 \mathrm{hrs}$ incubated (at $37^{\circ} \mathrm{C}$ ) culture of each microorganism was used. A serial dilution of the sample was prepared using sterile Mueller Hinton Broth medium containing $20 \mathrm{mM}$ Tris, $\mathrm{pH}$ 7, in 96 well sterile trays. Microorganisms were added to each well to $5 \times 10^{5} \mathrm{CFU} / \mathrm{ml}$ final concentration. Inoculated trays were then incubated for $20 \mathrm{~h}$ at $37^{\circ} \mathrm{C}$.

The bacterial growth was determined by plate reader instrument (Infinite 200 PRO NanoQuant Microplate Readers from Tecan, Switzerland) at $620 \mathrm{~nm}$. MIC values were expressed as the lowest concentration of peptide samples, reducing the growth by more than $50 \%{ }^{23}$. The MIC test was done in triplicate.

\section{Susceptibility test (Disc diffusion)}

Susceptibility test was carried out by the disc diffusion method ${ }^{24}$. Bacterial suspensions were prepared from overnight cultures in sterile normal saline and then adjusted to 0.5 McFarland standard turbidity test tubes. Then sterile cotton swabs were used for spreading of bacterial inoculums onto Mueller Hinton Agar (MHA) plates. The surface of the medium was allowed to be dried for about $3 \mathrm{~min}$. Sterile paper discs $(6 \mathrm{~mm}$ in diameter) were then placed onto the $\mathrm{MH}$ agar surface and $20 \mu \mathrm{l}$ of sample (from the stock of 1000 ìg/ml in 50-mmol/l Tris-HCl buffer, $\mathrm{pH}$ 7.4) was added per disc immediately. The discs with chloramphenicol (CHL, 30 ìg/disc) antimicrobial agent were used as standard antibiotic, and the blank disc containing $20 \mathrm{il}$, Tris-HCl buffer 50-mmol/ 
l, pH 7.4, served as a negative control. Plates were incubated at $37^{\circ} \mathrm{C}$ for $24 \mathrm{~h}$. Zones of inhibition were recorded in millimeters ${ }^{22}$.

\section{Agar-overlay assay}

After native electrophoresis $(20 \%$ polyacrylamide) of the sample, native gels were subjected to the agar overlay assay as described previously ${ }^{25}$. In this case, each gel slice was washed with $200 \mathrm{ml}$ of Milli-Q water for additional 4h, in room temperature. Each sliced gel was placed in a sterile petri dish and was covered with $7 \mathrm{ml}$ of $50^{\circ} \mathrm{C}$ melted Mueller-Hinton medium containing $0.75 \%$ agar and $5 \times 10^{7} \mathrm{CFU}$ of the test microorganism. Incubation was done at $35^{\circ} \mathrm{C}$ and $30^{\circ} \mathrm{C}$ for $20 \mathrm{~h}$. Escherichia coli and Staphylococcus aureus were assessed as Gram positive and Gram negative bacterial model, respectively. Developing of any zone of inhibition after this incubation period could reveal the presence of antibacterial proteins or peptides in the sample.

\section{Computational studies}

\section{Peptide modeling}

Pep-fold server was used for de-novo modeling of peptide structure from amino acid sequence using hidden Markov model-derived structural alphabet (http://bioserv.rpbs.univ-parisdiderot.fr/services/PEP-FOLD/). This server performs 200 simulations and generates clusters of models. Therefore the peptide sequence was submitted as input. Models are ranked according to sOPEP (Optimized Potential for Efficient structure Prediction) energy ${ }^{26}$. In order to achieve best structure, the CABS-fold ${ }^{27}$, Jpred $4{ }^{28}$, PSIPRED $^{29}$, Robetta ${ }^{30}$ and Raptor ${ }^{31}$ servers were also utilized and the resulting structures were compared and superimposed to predict the secondary and tertiary structure of this peptide. All servers done this job (de-novo modeling) in water matrix surrounding.

The best model with lowest SOPEP from pep-fold server was selected for further evaluation by subjecting it to 50.000 ps Molecular Dynamic Simulation using Gromacs 4.6.7 ${ }^{32}$.

\section{Molecular Dynamics Simulation}

All Molecular dynamics (MD) simulations were performed using the Groningen machine for chemical simulations (GROMACS) V 4.6.7 package $^{33}$. The Pep-fold server pdb atomic coordinate file was used for performing MD simulations. Systems were solvated in a cubic box of $1.2 \mathrm{~nm}$ distance (distance between the solute and the box) by applying AMBER99SB force field using the TIP3P water model. Particle-mesh Ewald (PME) electrostatic and periodic boundary conditions were applied. Neutralizing the system was performed through adding counter ion (CL') followed by replacing a water molecule in the system. Steepest descent energy minimization (2000 steps) was performed until the maximum force was smaller than $1000 \mathrm{KJ} / \mathrm{mol} / \mathrm{nm}$, to get rid of steric clashes and high-energy interactions [34]. Temperature adjustment was performed using NVT MD equilibration run for 50.000 ps at $300 \mathrm{~K}$. The resulting atom velocities and coordinates were used to start an NPT MD at $300 \mathrm{~K}$ and 1 bar for 500 ps by Parrinello-Rahman algorithm with tau- $\mathrm{t}=2.0$ ps, the density of the system was $986.61 \mathrm{~kg} / \mathrm{m}^{3}$ [35]. The production MD simulation in the course of 50,000 ps at constant pressure and temperature (1 bar and 300K) without position restraints was performed. A time step of 2 fs was used for the integration of equation of motion. All analysis of MD simulation was performed using GROMACS analysis tools. The values obtained for trajectory were averaged. The root mean square deviation (RMSD), Radius of gyration (Rg) and root mean square fluctuation (RMSF) and hydrophobic/ hydrophilic solvent accessible surface area (SAS) was followed throughout MD. The DSSP (hydrogen bond estimation algorithm) analysis was performed during 50,000 ps MD using DSSP 2.0.4 ${ }^{36}$.

\section{Calculation of Peptide electrostatics}

The resulting pdb structure was obtained by clustering last $10 \mathrm{~ns}$ of the simulation trajectory. The atomic charges of the molecule were assigned according to the partial charges of the AMBER99SB force field ${ }^{37}$, pKa values of titratable side chains were predicted by the Virginia Tech $\mathrm{H}++$ server (http://biophysics.cs.vt.edu/H++) at $\mathrm{pH} 7.0^{38,39}$, The electrostatic representation of the molecule was calculated using APBS 1.1 [40] and VMD 1.9.2 software (Visual Molecular Dynamics, Urbana, IL, USA).

\section{RESULTS}

\section{Peptide characterization}

SDS pages shows there are several proteins exist in early steps of the purification 
(Figure 2) and they must be purified in various steps. HPLC chromatogram is illustrated in Figure 3 nine protein fractions were observed in the extract after passing through $10 \mathrm{KD}$ centrifugal ultra-filters. Isolated peaks from RP-HPLC were further analysed using MALDI-TOF/TOF. The result of MS data submission to Mascot and NCBI database identified the presence of a small peptide "Dolabellanin B2" 41 .

Table 1. Minimum inhibitory concentrations (MICs) of fraction 8 against gram-negative and gram-positive bacteria by broth-dilution assay

\begin{tabular}{ccc}
\hline Microorganisms & $\begin{array}{c}\text { Gram-negative } \\
\text { or Gram-positive }\end{array}$ & $\mu \mathrm{g} / \mathrm{ml}$
\end{tabular}

\begin{tabular}{lcc}
\hline Staphylococcus aureus & + & 10 \\
Bacillus subtilis & + & 5 \\
Escherichia coli & - & 20 \\
Serratia marcescens & - & 10 \\
Klebsiella pneumoniae & - & 12.5 \\
Proteus mirabilis & - & 15 \\
Pseudomonas aeruginosa & - & 25
\end{tabular}

Table 2. demonstrates the antibacterial activity tested by the disc - diffusion method, inhibition zone values (in $\mathrm{mm}$ ) are presented as mean $\pm \mathrm{SD}$. Maximum and minimum inhibition zone diameter were observed against Bacillus subtilis and Pseudomonas aeruginosa respectively.

As shown in the Figure 4 identified peptide (fraction 8) developed a clear zone of inhibition in native gel, which was assessed against $S$. aureus and E. coli strains. The agar overlay assay is presented in Figure 4a: Escherichia coli plate, and 4b: Staphylococcus aureus plate. Total $20 \mu \mathrm{g}$ of purified peptide were loaded for each test.

\section{Peptide Modeling}

The structure obtained from Pep-fold (figure 5 green colored structure) was nearly superimposable with all other web-based methods, especially in the first alpha helix (amino acids 2-15) and the loop region (backbone RMSD of 0.8 - 1.2 A). The sOPEP of the model was -75.3 , which indicates peptide stability ${ }^{42}$. In addition, the template modeling score, tm value, was 0.42 that indicates the quality of peptide structure according the template alignments used ${ }^{43}$.

The CABS (C-Alpha, C-Beta, Side-chain) algorithm is one of the most efficient tools for

\section{Antibacterial activity}

As it is shown in Table 1. The purified peptide showed strong antimicrobial activity against strains of gram positive and gram negative bacteria. The susceptibility of the bacteria against dolabelanin-B2 was: Bacillus subtilis> Staphylococcus aureus > Serratia marcescens> Klebsiella pneumoniae $>$ Proteus mirabilis $>$ Escherichia coli> Pseudomonas aeruginosa.

Table 2. Disk-diffusion assay.

The values are presented as mean \pm SD

\section{Microorganisms \\ (Gram-negative and Gram-positive)}

$100 \mu$ g peptide per $6 \mathrm{~mm}$ disk on MHA plate (mm)

$\begin{array}{lc}\text { Staphylococcus aureus } & 21.5 \pm 0.5 \\ \text { Bacillus subtilis } & 25.0 \pm 1.5 \\ \text { Escherichia coli } & 11 \pm 0.0 \\ \text { Serratia marcescens } & 19.5 \pm 1.3 \\ \text { Klebsiella pneumoniae } & 16.5 \pm 0.5 \\ \text { Proteus mirabilis } & 14 \pm 0.5 \\ \text { Pseudomonas aeruginosa } & 9 \pm 1.0\end{array}$

protein structure prediction and has demonstrated accurate structure predictions for difficult de novo (or unclearly homological) targets ${ }^{27}$. Interestingly, the CABS-fold server output was very similar to our simulation result except for some side chain rotations and therefore provides closest structure to our 50ns molecular dynamics simulation (figure 5 gray colored structure). The CABS simulation temperature, energy and radius of gyration were 3.5-1.0, -350 and $1 \mathrm{~nm}$ after 400 CABS MC (Monte Carlo) microcycles ${ }^{44}$. The model produced by Robetta showed two extended and well-formed alpha helixes and hence the smaller loop region (figure 5 red colored). Raptorx server prediction, colored blue in figure 5 similarly superimposes well with others in the first helix but shows unstructured conformation for residues 21-30 and only forms a small helical structure in the c- terminal region.

\section{Molecular dynamics}

The molecular dynamics simulation was performed for 50,000 ps. We investigated a root mean square deviation (RMSD) of the backbone atoms that is a measure of distance between different spatial structures. Time evolution of the peptide RMSD is shown in Figure 6a. As demonstrated, process of structure formation 


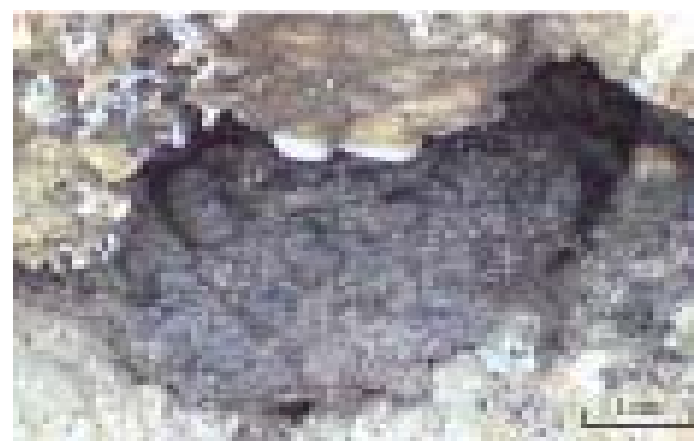

Fig. 1. Marine gastropod, Peronia peronii on rocky shore in the Queshm Island, the Persian Gulf

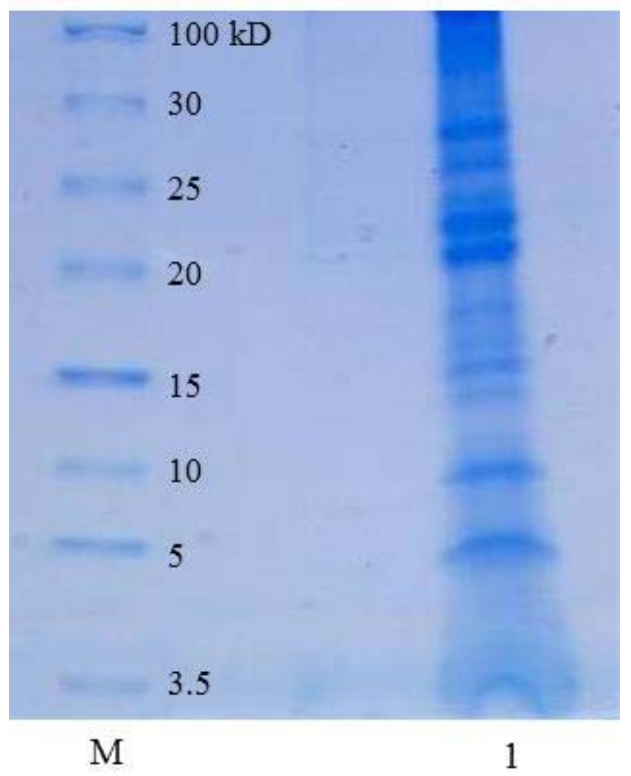

Fig. 2. Electrophoresis analysis of Peronia peronii, under 100KD protein extraction (lane 1). Thermo scientific Protein Ladder (lane M), 5 to 25\% gradient SDS-page

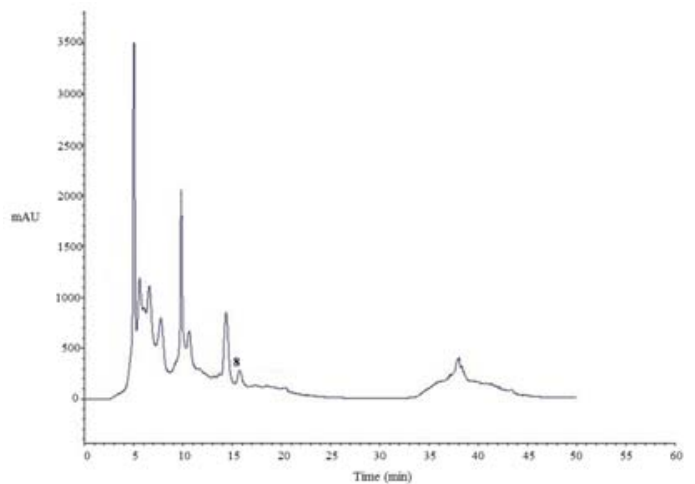

Fig. 3. Chromatogram of proteins after $10 \mathrm{KD}$ ultrafiltration by RP-HPLC; number 8 adjacent to the fraction with antibacterial, $\mathrm{mAU}=$ mill absorbance units occurred in the first 1 ns of trajectory that is expressed in the change of RMSD from $0.1 \mathrm{~nm}$ to nearly constant value of $0.25 \pm 0.032 \mathrm{~nm}$ in the next 10 ns of simulation. The backbone RMSD for the last 10 ns was less than $0.1 \mathrm{~nm}$.

Figure 6b. shows the radius of gyration (Rg, indicator of protein structure compactness) during the simulation time ${ }^{45}$, as it can be inferred the overall spread of the molecule remained at 1.01 $\pm 0.025 \mathrm{~nm}$. Therefore, it seems that a stable structure obtained after 50 ns MD. Hence, the obtained trajectory would be suitable for subsequent calculations.

Figure 7. demonstrates the secondary structure for each residue in the course of 50,000 ps simulation, as it could be understood form DSSP graph, the secondary structure content varies in the first 40 ns of MD simulation. These alterations between alpha-helix and 3-helix are related to residues 23-30, which gradually shifted to a stable alpha helical structure, colored blue in the DSSP graph. The helixes are connected with bend (green) and coil forming residues 15-21, which is likely attributed to the presence of Proline 19. The secondary structure elements, which were assigned by DSSP algorithm, matched with rigid regions having lowest RMSF values (data not shown). Cluster analysis was performed to identify conformational states that were actually visited during the MD. The cluster cutoff used to ensure that the root mean square deviation (RMSD) in a single cluster was less than $0.10 \mathrm{~nm}$. The resulting structure (Found 1. cluster) is presented in Figure 8a using VMD.

\section{Peptide surface electrostatics and hydrophobicity}

The electrostatic potential surface around the modeled peptide is shown in Figure 8b Variations in the electrostatic fields surrounding the peptides are caused by alterations in the local charge and charge distribution. The total charge of the Dolabelanin-B2 peptide was calculated to be $Z=1$. The potential field of the Dolabelanin-B2 peptide consists of the dominant positive lobe (main contributors are Lys 11, 18 and 25 which are scattered along the peptide). While, the negative lobes are appeared in the $\mathrm{N}$-terminal, which are attributed to the presence of two spatially close residues Asp 4 and Glu 7, the only negatively charged residues in the sequence (Figure $8 \mathrm{~b}$ ). The distribution of positive charge contributes to the 
high-volume positive coulomb cage bulb around the peptide. Isosurface value units are $\mathrm{kT} / \mathrm{e}=25$ $\mathrm{mV}$ at room temperature. Together with charge distribution the surface area of a biomolecule that is accessible to a solvent play important role in protein function and stability ${ }^{46}$. Figure 9 shows the solvent accessible surface area (SASA) change

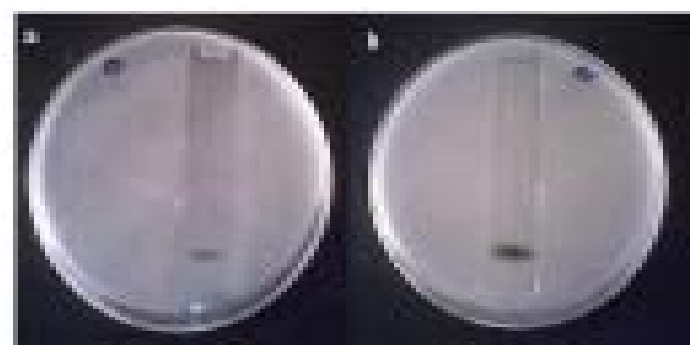

Fig. 4. Agar overlay assay Panel a: Escherichia coli and panel b: Staphylococcus aureus plates

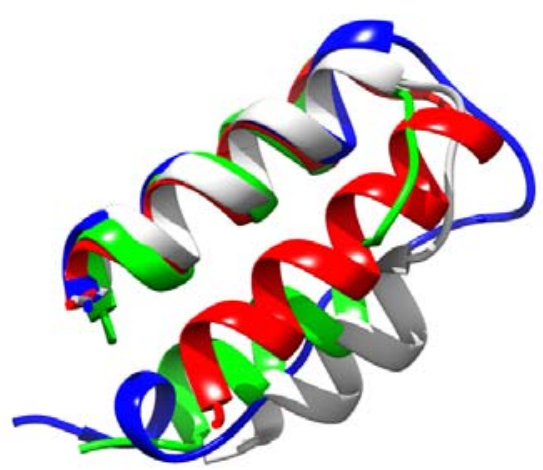

Fig. 5. NewCartoon representation of superimposed models predicted by PEP-Fold (green), Robeta (red), CABS (light gray) and Raptorx (blue). The n-terminal helix is more intact in different methods. over simulation time, the total surface area (30.5 \pm $1.28 \mathrm{~nm} 2$ ) was obtained by Gromacs tools, and the area of the molecule that is in contact with water molecules network around. Hydrophobic and hydrophilic surfaces of the molecule were $13.96 \pm$ $0.68 \mathrm{~nm}^{2}$ and $16.55 \pm 0.88 \mathrm{~nm}^{2}$, respectively.
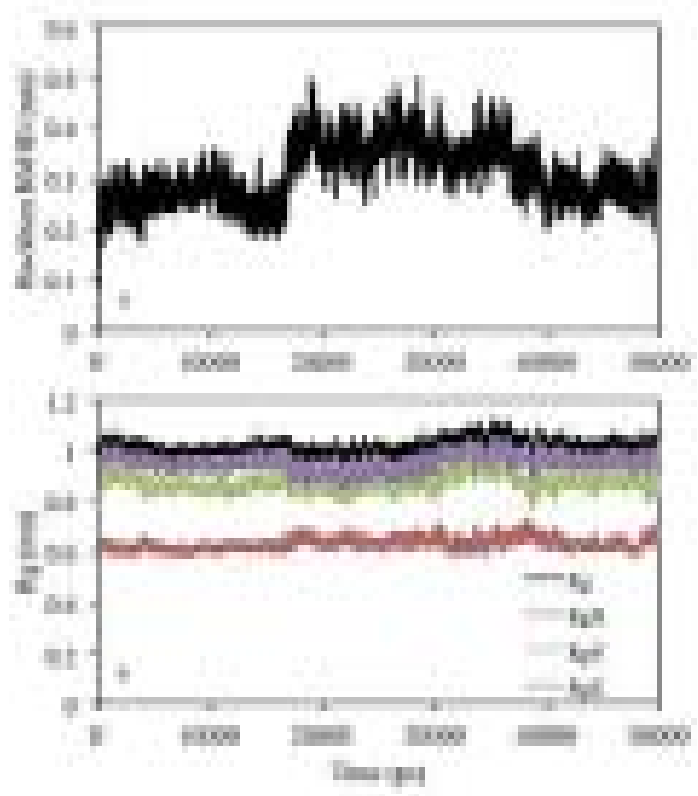

Fig. 6. The RMSD (plan a) and radius of gyration (plan b) analysis as indicative of the level of compaction and stability of simulated structure. Both graphs start with sharp changes at the beginning, but gradually reach a steady state region with small fluctuations

\section{Secondary structure}

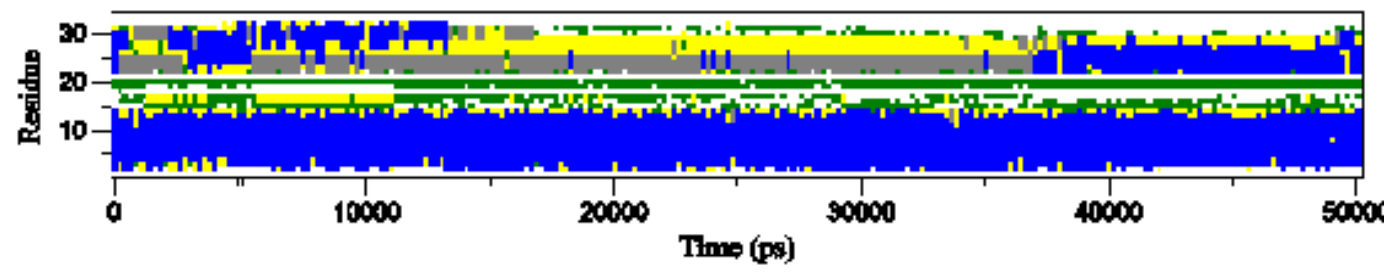

\section{$\square$ Coil $\square$ Bend $\square$ Tum $\square$ A-Helix $\square$ 3-Helix}

Fig. 7. DSSP plot of secondary structure of Dolabellanin-B2 as a function of time in a simulated system. Secondary structure elements; coil (white), bend (green), turn (yellow), alpha-helix (blue) and 3-helix (gray) for each residue is shown 

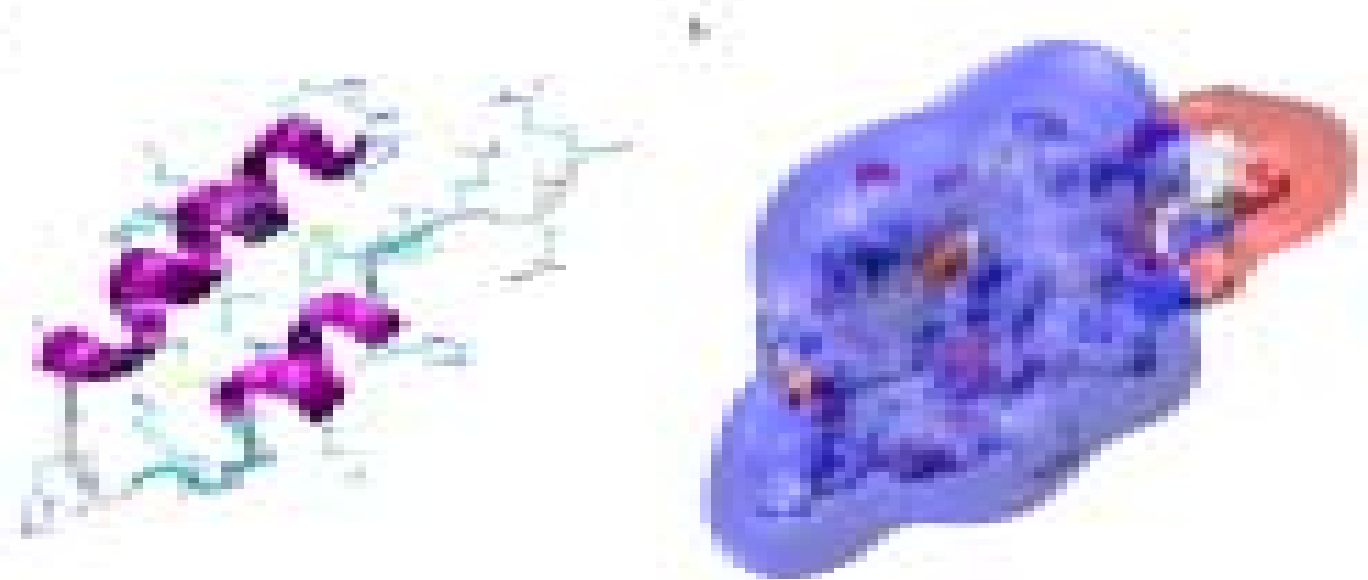

Fig. 8 (a) The carton structure of simulated Dolabellanin-B2 colored by VMD secondary structure, the amino acid side chains also showed in line mode colored by element type, disulfide bridges are displayed in yellow. b) The electrostatic potential surface around the peptide, the Connolly surface is colored by charge, while their positive and negative residues are drawn in blue and red, respectively. The Coulomb cages for the positive (transparent blue) and negative (transparent red) domains are drawn in the distance where the electrostatic potential equals $1 \mathrm{k}_{\mathrm{B}} \mathrm{T} / \mathrm{e}$

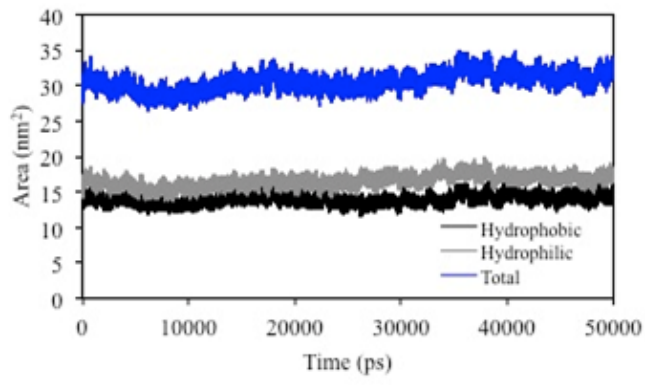

Fig. 9. hydrophilicity and hydrophobicity terms of solvent accessible surface area of simulated Dolabellanin-B2 obtained during the entire period of simulation the average of the values were $13.96 \pm$ $0.54 \mathrm{~nm}^{2}$ for hydrophobic surface (black) and 16.22 $\pm 0.66 \mathrm{~nm}^{2}$, for Hydrophilic (gray.

\section{DISCUSSION}

The production of antimicrobial peptides is a widespread mechanism of host defense in the living organisms, from bacteria, protozoans, and invertebrates to vertebrates and plants. The antimicrobial peptides grouped into distinct families according to common features of primary and secondary structures ${ }^{47}$.

Lijima and coworkers introduced a small peptide (3872.5 Da) from marine mollusk, Dolabella auricularia, called Dolabellanin-B2. They reported the Dolabellanin B2 sequence (SHQDCYEALHK CMASHSKPFSCSMKFHMCLQQQ) and also declared that this peptide has an antibacterial and antifungal activity ${ }^{41}$. According to mass spectra in addition to the antimicrobial peptide database (APD, http://aps.unmc.edu/AP) our purified peptide most probably poses the similar sequence. For our searched query the retrieved result is APD ID: AP00284, Unknown 3D Structure and found in sea hare, Dolabella auricularia. Which is currently classified under "mollusks" peptide family with another 31 identified sequences ${ }^{48}$. The presence of similar antibacterial peptide in these two species could be representative of the important function of this peptide. Marine gastropods, Dolabella auricularia in Lijima and coworkers and Peronia peronii in this study belongs to subclass Heterobranchia.

Characterization of protein and peptide structures is an important subject to understand their functions. Many of studies shown peptide properties like amino acids composition, peptide length, molecular weight, isoelectric point, net charge, hydrophobicity, hydrophobic arc size and amphiphilicity have undeniable roles and able to influence the ability of alpha CAMPs to insert into microbial membranes and kill target microbes ${ }^{49}$. While X-ray and NMR are most reliable techniques in structural studies, Computational methods like homology, threading, and ab initio modeling are nowadays fast and applicable for predicting and exploring $3 \mathrm{D}$ properties of a given protein 
sequence $^{50}$. Peptide tertiary structure constitutes a basis to better rationalize further exploration by indicating the role of specific positions responsible for the peptide activity. Despite particular progress using NMR spectroscopy, the number of peptide structures experimentally solved remains low. According to APD database there are 1341 entries with unknown structures among 2,121 antibacterial peptides, in which 412 of them are only Anti-G ${ }^{+}$ and the number of only Anti-G peptides are 197. The alpha-helical family is the largest with 335 members compare with 76 and 59 for beta and combine alpha/beta structures, respectively.

For proteins, the best option to get an in silico 3D model is homology modeling. The homology modeling requires a determined structure, which must be homologous (sequence identity usually greater than 30\%) to the input sequence. PSI-BLAST was used to find the best templates for homology modeling, but no results were returned for the input sequence. Alternatively, data mining in a non-redundant database CAMP, Collection of Antimicrobial Peptides ${ }^{51}$ highlighted a template 1E4T with 30\% identity, possessing 37 residues and three disulfide bonds. 1EAT is a Beta defensin from Mus musculus (Mouse). It should be mentioned that, there are two main concerns using comparative modeling; first, the sequences should be homologous and as a result we expect similar topology. Second, lower the sequence identity there would be the larger error on the modeling ${ }^{52}$. Therefore, this approach faces several limitations that prevent their use for small peptides, which are in overall more flexible and sensitive to substitutions, especially when there is no template with high identity. Therefore, the retrieved structure could not be a suitable template due to low identity considering the sequence size. So as we reached mostly similar models from different modeling servers, we used Pep-fold structure (as a specific for peptide with de-novo modeling) as a starting structure for our further evaluation.

Molecular dynamics (MD) simulation techniques need high performance computation. Hence, knowledge-based shortcuts learned from the presence of conformations (loops, beta turns etc.) or prediction of secondary structure has been specially designed for peptides. These methods are faster than MD simulations and are available via on-line servers. The PEP-FOLD server is a peptide specific approach, which relies on a more accurate description of local conformation (structural alphabet), using 27 different canonical states and geometrical descriptions ${ }^{53}$.

Peptides tend to be more flexible than proteins and dynamic personality of peptides would play a greater role in their properties. Thus, the main aspect of peptide modeling is to analyze the trajectory outcome of an MD simulation. Therefore, here we used both above approaches to predict the tertiary structure of DolabellaninB2. As Figure 8a demonstrates, residues 8 (Ala) to 30 (Leu) are positively charged (Lys 11, 18 and 25) or hydrophobic, interrupted by the presence of four polar serines (15, 17, 21 and 23) while negatively charged residues are in the N-terminal (4 and 7) therefore, leading to the charge discrimination (Figure 8b, note the positioning of Lys residues). However, the histidine residues (2, 10, 16 and 27) could affect the antibacterial activity by adding partial positive charge in the acidic condition [54]. The presence of highly hydrophilic positively charged region could account for high electrostatic affinity to Lipopolysaccharide (LPS) and the presences of positively charged residues in the hydrophobic area can impede further penetration of the peptide into the lipid bilayer. Separation of hydrophobic and hydrophilic regions possibly could have enabled peptide against Gram-negative bacteria. It is shown that antimicrobial peptides need an optimal "hydrophobicity threshold" for insertion into zwitterionic micellar membranes [55] and hydrophobic interactions are essential for the membrane 'sinking' process after the initial attachment ${ }^{56}$. Therefore, active antimicrobial peptides must have an appropriate balance of hydrophobicity and net positive charge ${ }^{57}$.

The obtained model has similarities with a novel fold antimicrobial peptide, Ec-AMP1 (PDB: 2L2R, 37 amino acids, antifungal peptide from kernels of barnyard grass Echinochloa crusgalli). Ec-AMP1 is a cationic á-helical hairpin structure held together by a pair of nested disulfide bonds [58]. Interestingly, Glycocin F glycopeptide (PDB: 2KUY, 43 a.a. bacteriocin from Lactobacillus plantarum) shows a similar hairpin fold ${ }^{59}$. The Bacillus subtilis strain 168 produces the extremely stable antibiotic sublancin 168 , which is a $37-$ residue peptide with the similar helix-loop-helix structure (entry 2MIJ) ${ }^{60}$. Similarly, Monincova et 
al. declared that lasiocepsin (PDB: 2MBD, Lasioglossum laticeps, wild bee venom) is an alpha helical peptide consists of 27 residues, and shares no significant sequence homology with known AMPs or other proteins annotated in the public databases (APD or UniProtKB) ${ }^{61,62}$. Meanwhile, lasiocepsin poses substantial antibacterial and antifungal activity (tested against $B$. subtilis, $S$. aureus, E. coli, P. aeruginosa and C. albicans) consists of two vertically positioned unequal alpha helixes held together by two disulfide bonds. Lasiocepsin were shown to be capable of permeabilising the bacterial cell membrane and penetrates both the outer and inner membranes of Gram-negative bacteria ${ }^{62}$. Therefore, we can suggest that the hairpin structure of DolabelaninB2 could possibly penetrate cell membrane as well.

The electrostatic fields of the Antimicrobial peptides may suggest that electrostatic forces play a key role in the peptidemembrane interactions ${ }^{63}$. This discrimination in electrostatic potentials around the peptide (as shown in Figure 8b) could possibly determine the orientation and interaction mechanism of cationic AMPs. Transmembrane potential and interaction with acidic moieties of lipid A together with hydrophobic interaction with the hydrocarbon chain stabilizes the AMP interaction and hence destabilizes membrane integrity and in consequence functions. In addition, the interaction of AMP with $\mathrm{Ca}^{+2}$ and $\mathrm{Mg}^{+2}$ (as membrane stabilizers by crosslinking phospholipid head groups) affects membrane stability ${ }^{49}$. It should be noted that, in addition to membranes, AMPs could bind to DNA, heat shock proteins, carbohydrates, and lipids and therefore act in different ways ${ }^{64}$.

Most of the naturally occurring AMPs are not optimized for efficient activity and need to be improved through various strategies, before it would be used as therapeutics. Recently, different methods have been tested using the native templates to generate more efficient AMPs such as quantitative structure-activity relationship (QSAR), random mutagenesis, altering the peptide structures by cyclization, or increasing the charge or hydrophobicity of the peptide by tagging ${ }^{65}$. The study revealed that cationicity alone is not the determining factor in the microbicidal activity of antimicrobial peptides. A molecular dynamics study by Zhao et al. (2014) shown the formation of structural domains in bacterial membrane as a result of reorganization and the localization of anionic lipids in response to the binding of cationic antimicrobial agents (HBD-3) [66]. Factors affecting the molecular dynamics such as hydrophobicity, electrostatic interactions and the potential for oligomerization may also play fundamental roles. It points to the usefulness of MD simulation studies in successful engineering of antimicrobial peptides for improved activity ${ }^{67}$. Therefore, a number of parameters modulate the antimicrobial activity, including net positive charge, charge distribution, amphipathicity, and helical propensity.

\section{CONCLUSION}

Marine invertebrates have developed an effective nonspecific immune system to defend against pathogenic attack by microorganisms. In this work we applied the methods of molecular dynamics simulation and antibacterial assays to give insights into spatial structure and possible mechanism of action for previously identified natural antimicrobial peptide Dolabelanin-b2. Molecular properties such as secondary structure content, surface hydrophobicity and electrostatic potential distribution suggest that probably this peptide is related to alpha-helical cationic AMPs. Biophysical analysis of peptides with computational and experimental methods may also shed light on the exact roles of various parameters, such as net charge, percent of á-helical/â-sheet and amphipathicity, on the ability and mechanism of antimicrobials. Marine invertebrate antimicrobial peptides together with molecular dynamics studies need more efforts and hopefully provide the opportunity for a breadth of research on new natural antibiotics.

\section{REFERENCES}

1. Duraikannu, K., et al., Antimicrobial peptide from Marine Sponge Clathria indicqa. American Eurasian Journal of Scientific Research, 2009. 4: p. 47-53.

2. Faulkner, J., Marine natural products. Natural Product Reports, 1996. 13: p. 75-125.

3. Abad, M.J., Bedoya, L. M., Bermejo, P., Marine Compounds and their Antimicrobial Activities. Science against microbial pathogens: communicating current research and 
technological advances, 2011: p. 14.

4. Abad, M.J., L.M. Bedoya, and P. Bermejo, Natural marine anti-inflammatory products. Mini Rev Med Chem, 2008; 8(8): p. 740-54.

5. Bonomo, R.A., Multiple antibiotic-resistant bacteria in long-term-care facilities: An emerging problem in the practice of infectious diseases. Clin Infect Dis, 2000; 31(6): p. 1414-22.

6. Zasloff, M., Antimicrobial peptides of multicellular organisms. Nature, 2002; 415(6870): 389-95.

7. Otero-Gonzalez, A.J., et al., Antimicrobial peptides from marine invertebrates as a new frontier for microbial infection control. FASEB J, 2010; 24(5): p. 1320-34.

8. Boman, H.G., Peptide antibiotics and their role in innate immunity. Annu Rev Immunol, 1995; 13: p. 61-92.

9. Brogden, K.A., Antimicrobial peptides: pore formers or metabolic inhibitors in bacteria? Nat Rev Microbiol, 2005; 3(3): p. 238-50.

10. Matsuzaki, K., Why and how are peptide-lipid interactions utilized for self-defense? Magainins and tachyplesins as archetypes. Biochimica et Biophysica Acta (BBA) - Biomembranes, 1999; 1462(1-2): p. 1-10.

11. Tincu, J.A. and S.W. Taylor, Antimicrobial peptides from marine invertebrates. Antimicrob Agents Chemother, 2004; 48(10): p. 3645-54.

12. Rodriguez, J., R. Riguera, and C. Debitus, The natural polypropionate-derived esters of the mollusk Onchidium sp. The Journal of Organic Chemistry, 1992; 57(17): p. 4624-4632.

13. Sharadadevi, A. and R. Nagaraj, A molecular dynamics study of human defensins HBD-1 and HNP-3 in water. J Biomol Struct Dyn, 2010; 27(4): p. 541-50.

14. Dayrat, B. and S. Tillier, Evolutionary relationships of euthyneuran gastropods (Mollusca): a cladistic re-evaluation of morphological characters. Zoological Journal of the Linnean Society, 2002; 135(4): p. 403-470.

15. Dayrat, B., Review of the current knowledge of the systematics of Onchidiidae (Mollusca: Gastropoda: Pulmonata) with a checklist of nominal species. Zootaxa, 2009; 2068.

16. Jang, W.S., et al., Halocidin: a new antimicrobial peptide from hemocytes of the solitary tunicate, Halocynthia aurantium. FEBS Lett, 2002; 521(13): p. 81-6.

17. Ovchinnikova, T.V., et al., Aurelin, a novel antimicrobial peptide from jellyfish Aurelia aurita with structural features of defensins and channel-blocking toxins. Biochem Biophys Res Commun, 2006; 348(2): p. 514-23.

18. Lee, I.H., et al., Dicynthaurin: an antimicrobial peptide from hemocytes of the solitary tunicate, Halocynthia aurantium. Biochim Biophys Acta, 2001; 1527(3): p. 141-8.

19. Lehrer, R.I., et al., Clavanins and styelins, alphahelical antimicrobial peptides from the hemocytes of Styela clava. Adv Exp Med Biol, 2001; 484: p. 71-6.

20. Sparbier, K., et al., Analysis of glycoproteins in human serum by means of glycospecific magnetic bead separation and LC-MALDI-TOF/TOF analysis with automated glycopeptide detection. J Biomol Tech, 2007; 18(4): p. 252-8.

21. Rehbein, P. and H. Schwalbe, Integrated protocol for reliable and fast quantification and documentation of electrophoresis gels. Protein Expr Purif, 2015; 110: p. 1-6.

22. Jorgensen, T., Antibacterial susceptibility tests: dilution and disk diffusion methods. Manual of Clinical Microbiology, seventh ed. ASM Press, Washington, DC, 1999; 1526-1543.

23. Perumal Samy, R., et al., In vitro antimicrobial activity of natural toxins and animal venoms tested against Burkholderia pseudomallei. BMC Infect Dis, 2006; 6: 100.

24. Bauer, A.W., et al., Antibiotic susceptibility testing by a standardized single disk method. Am J Clin Pathol, 1966; 45(4): p. 493-6.

25. S.K., K. and A. C., Bacteriocin production by Lactococcus lactis KCA 2386 isolated from white kimchi. Food Science and Biotechnology, 2000; 9: 263-296.

26. Thevenet, P., et al., PEP-FOLD: an updated de novo structure prediction server for both linear and disulfide bonded cyclic peptides. Nucleic Acids Res, 2012; 40(Web Server issue): p. W28893.

27. Blaszczyk, M., et al., CABS-fold: server for the de novo and consensus-based prediction of protein structure. Nucleic Acids Research, 2013; 41(W1): W406-W411.

28. Drozdetskiy, A., et al., JPred4: a protein secondary structure prediction server. Nucleic Acids Res, 2015.

29. Buchan, D.W., et al., Scalable web services for the PSIPRED Protein Analysis Workbench. Nucleic Acids Res, 2013; 41(Web Server issue): p. W349-57.

30. Kim, D.E., D. Chivian, and D. Baker, Protein structure prediction and analysis using the Robetta server. Nucleic Acids Res, 2004; 32(Web Server issue): p. W526-31.

31. Källberg, M., et al., Template-based protein structure modeling using the RaptorX web server. Nat. Protocols, 2012; 7(8): p. 1511-1522.

32. Hess, B., et al., GROMACS 4: Algorithms for Highly Efficient, Load-Balanced, and 
Scalable Molecular Simulation. Journal of Chemical Theory and Computation, 2008; 4(3): p. 435-447.

33. Pronk, S., et al., GROMACS 4.5: a highthroughput and highly parallel open source molecular simulation toolkit. Bioinformatics, 2013.

34. Van Der Spoel, D., et al., GROMACS: fast, flexible, and free. J Comput Chem, 2005; 26(16): p. 1701-18.

35. Parrinello, M. and A. Rahman, Polymorphic transitions in single crystals: A new molecular dynamics method. Journal of Applied Physics, 1981; 52(12): p. 7182-7190.

36. Kabsch, W. and C. Sander, Dictionary of protein secondary structure: pattern recognition of hydrogen-bonded and geometrical features. Biopolymers, 1983; 22(12): p. 2577-637.

37. Hornak, V., et al., Comparison of multiple Amber force fields and development of improved protein backbone parameters. Proteins, 2006; 65(3): p. 712-25.

38. Anandakrishnan, R., B. Aguilar, and A.V. Onufriev, $\mathrm{H}++$ 3.0: automating $\mathrm{pK}$ prediction and the preparation of biomolecular structures for atomistic molecular modeling and simulations. Nucleic Acids Research, 2012; 40(W1): p. W537-W541.

39. Myers, J., et al., A simple clustering algorithm can be accurate enough for use in calculations of pKs in macromolecules. Proteins: Structure, Function, and Bioinformatics, 2006; 63(4): p. 928-938.

40. Baker, N.A., et al., Electrostatics of nanosystems: application to microtubules and the ribosome. Proc Natl Acad Sci U S A, 2001; 98(18): p. 10037-41.

41. Iijima, R., J. Kisugi, and M. Yamazaki, A novel antimicrobial peptide from the sea hare Dolabella auricularia. Dev Comp Immunol, 2003; 27(4): p. 305-11.

42. Maupetit, J., P. Derreumaux, and P. Tuffery, A fast method for large-scale de novo peptide and miniprotein structure prediction. J Comput Chem, 2010; 31(4): p. 726-38.

43. Zhang, Y. and J. Skolnick, Scoring function for automated assessment of protein structure template quality. Proteins, 2004; 57(4): p. 70210.

44. Blaszczyk, M., et al., CABS-fold: Server for the de novo and consensus-based prediction of protein structure. Nucleic Acids Res, 2013; 41(Web Server issue): p. W406-11.

45. Lobanov, M.Y., N.S. Bogatyreva, and O.V. Galzitskaya, Radius of gyration as an indicator of protein structure compactness. Molecular
Biology, 2008; 42(4): p. 623-628.

46. Lee, B. and F.M. Richards, The interpretation of protein structures: estimation of static accessibility. J Mol Biol, 1971; 55(3): p. 379400.

47. He'tru, C., D. Hoffmann, and P. Bulet, Antimicrobial peptides from insects. Molecular Mechanisms of Immune Responses in Insects, 1998; 40-60.

48. Wang, G., X. Li, and Z. Wang, APD2: the updated antimicrobial peptide database and its application in peptide design. Nucleic Acids Res, 2009; 37(Database issue): p. D933-7.

49. Phoenix, D.A., Dennison, S. R. and Harris, F., Antimicrobial Peptides, ed. first. 2013: WileyVCH Verlag GmbH \& Co. KGaA. 221.

50. Beaufays, J., et al., In silico predictions of 3D structures of linear and cyclic peptides with natural and non-proteinogenic residues. $J$ Pept Sci, 2012; 18(1): p. 17-24.

51. Waghu, F.H., et al., CAMP: Collection of sequences and structures of antimicrobial peptides. Nucleic Acids Res, 2014; 42(Database issue): p. D1154-8.

52. Marti-Renom, M.A., et al., Comparative protein structure modeling of genes and genomes. Annu Rev Biophys Biomol Struct, 2000; 29: p. 291325.

53. Thévenet, P., et al., De Novo Peptide Structure Prediction: An Overview, in Computational Peptidology, P. Zhou and J. Huang, Editors., Springer New York. 2015; 1-13.

54. Kacprzyk, L., et al., Antimicrobial activity of histidine-rich peptides is dependent on acidic conditions. Biochimica et Biophysica Acta (BBA) - Biomembranes, 2007; 1768(11): p. 2667-2680.

55. Stark, M., L.P. Liu, and C.M. Deber, Cationic hydrophobic peptides with antimicrobial activity. Antimicrob Agents Chemother, 2002; 46(11): p. 3585-90.

56. Glukhov, E., et al., Basis for selectivity of cationic antimicrobial peptides for bacterial versus mammalian membranes. J Biol Chem, 2005; 280(40): 33960-7.

57. Kluver, E., K. Adermann, and A. Schulz, Synthesis and structure-activity relationship of beta-defensins, multi-functional peptides of the immune system. J Pept Sci, 2006; 12(4): p. 24357.

58. Nolde, S.B., et al., Disulfide-stabilized helical hairpin structure and activity of a novel antifungal peptide EcAMP1 from seeds of barnyard grass (Echinochloa crus-galli). J Biol Chem, 2011; 286(28): p. 25145-53.

59. Wang, G., Improved methods for classification, prediction, and design of antimicrobial peptides. 
Methods Mol Biol, 2015; 1268: p. 43-66.

60. Garcia De Gonzalo, C.V., et al., NMR structure of the S-linked glycopeptide sublancin 168. ACS Chem Biol, 2014; 9(3): p. 796-801.

61. Monincova, L., et al., Lasiocepsin, a novel cyclic antimicrobial peptide from the venom of eusocial bee Lasioglossum laticeps (Hymenoptera: Halictidae). Amino Acids, 2012; 43(2): p. 75161.

62. Monincova, L., et al., Structural basis for antimicrobial activity of lasiocepsin. Chembiochem, 2014; 15(2): 301-8.

63. Ramamoorthy, A., et al., Solid-state NMR investigation of the membrane-disrupting mechanism of antimicrobial peptides MSI-78 and MSI-594 derived from magainin 2 and melittin. Biophys J, 2006; 91(1): p. 206-16.
64. Otvos, L., Jr., et al., Interaction between heat shock proteins and antimicrobial peptides. Biochemistry, 2000; 39(46): p. 14150-9.

65. Pushpanathan, M., P. Gunasekaran, and J. Rajendhran, Antimicrobial peptides: versatile biological properties. Int J Pept, 2013; 675391.

66. Zhao, X., et al., Simulating the antimicrobial mechanism of human beta-defensin-3 with coarse-grained molecular dynamics. J Biomol Struct Dyn, 2015: p. 1-8.

67. Soman, S.S., K.C. Sivakumar, and E. Sreekumar, Molecular dynamics simulation studies and in vitro site directed mutagenesis of avian betadefensin Apl_AvBD2. BMC Bioinformatics, 2010. 11 Suppl 1: p. S7. 